\title{
Preparation and characterization of monomethoxy poly(ethylene glycol)-poly( $\varepsilon$-caprolactone) micelles for the solubilization and in vivo delivery of luteolin
}

This article was published in the following Dove Press journal:

International Journal of Nanomedicine

12 August 2013

Number of times this article has been viewed

\author{
Jin-Feng Qiu' \\ Xiang Gao, ${ }^{1,2}$ \\ Bi-Lan Wang' \\ Xia-Wei Wei' \\ Ma-Ling Gou' \\ Ke Men' \\ Xing-Yu Liu' \\ Gang Guo' \\ Zhi-Yong Qian' \\ Mei-Juan Huang' \\ 'Department of Thoracic Oncology, \\ State Key Laboratory of Biotherapy \\ and Cancer Center, West China \\ Hospital and Medical School, Sichuan \\ University, Chengdu, People's \\ Republic of China; ${ }^{2}$ Medical School \\ and Department of Pathophysiology, \\ College of Preclinical and Forensic \\ Medical Sciences, Sichuan University, \\ Chengdu, People's Republic of China
}

Correspondence: Mei-Juan Huang; Ma-Ling Gou

Department of Thoracic Oncology, State Key Laboratory of Biotherapy and Cancer Center,West China Hospital and Medical School, Sichuan University, 37 Guo Xue Xiang, Chengdu 61004I, People's Republic of China

Email.hmj107@I63.com; basad@I63.com

\begin{abstract}
Luteolin (Lu) is one of the flavonoids with anticancer activity, but its poor water solubility limits its use clinically. In this work, we used monomethoxy poly(ethylene glycol)-poly( $\varepsilon$-caprolactone) (MPEG-PCL) micelles to encapsulate Lu by a self-assembly method, creating a water-soluble Lu/MPEG-PCL micelle. These micelles had a mean particle size of $38.6 \pm 0.6 \mathrm{~nm}$ (polydispersity index $=0.16 \pm 0.02$ ), encapsulation efficiency of $98.32 \% \pm 1.12 \%$, and drug loading of $3.93 \% \pm 0.25 \%$. Lu/MPEG-PCL micelles could slowly release $\mathrm{Lu}$ in vitro. Encapsulation of Lu in MPEG-PCL micelles improved the half-life $\left(t_{1 / 2} ; 152.25 \pm 49.92\right.$ versus [vs] $7.16 \pm 1.23$ minutes, $\left.P=0.007\right)$, area under the curve $(0-\mathrm{t})(2914.05 \pm 445.17$ vs $502.65 \pm 140.12 \mathrm{mg} / \mathrm{L} /$ minute, $P=0.001)$, area under the curve $(0-\infty)(2989.03 \pm 433.22$ vs $503.81 \pm 141.41 \mathrm{mg} / \mathrm{L} /$ minute, $P=0.001)$, and peak concentration $(92.70 \pm 11.61 \mathrm{vs} 38.98 \pm 7.73 \mathrm{mg} / \mathrm{L}, P=0.003)$ of $\mathrm{Lu}$ when the drug was intravenously administered at a dose of $30 \mathrm{mg} / \mathrm{kg}$ in rats. Also, Lu/MPEG-PCL micelles maintained the cytotoxicity of $\mathrm{Lu}$ on $4 \mathrm{~T} 1$ breast cancer cells ( $\mathrm{IC} 50=6.4 \pm 2.30 \mu \mathrm{g} / \mathrm{mL}$ ) and C-26 colon carcinoma cells $(\mathrm{IC} 50=12.62 \pm 2.17 \mu \mathrm{g} / \mathrm{mL})$ in vitro. These data suggested that encapsulation of Lu into MPEG-PCL micelles created an aqueous formulation of $\mathrm{Lu}$ with potential anticancer effect.
\end{abstract}

Keywords: luteolin, micelle, MPEG-PCL, cancer therapy

\section{Introduction}

Cancer is the leading cause of death in developed countries and the second-leading cause of death in developing countries. ${ }^{1}$ As the incidence of cancer is increasing, considerable attention is being paid to the prevention and therapy of cancer. Nonetheless, there are still many challenges in cancer therapy, and developing novel anticancer drugs is of interest. Natural products derived from plants play an important role in the health care of many cultures, both ancient and modern. ${ }^{2-4}$ Presently, over 100 new natural products are in clinical development, in particular anticancer and anti-infective agents. ${ }^{4}$ Natural compounds with antitumor activity and limited toxicity are getting more and more attention in medical research. Luteolin (Lu; 3',4',5',7'-tetrahydroxyflavone, shown in Figure 1A) belongs to a group of natural compounds named flavonoids that are found widely in the plant kingdom. ${ }^{5}$ As with other flavonoids, $\mathrm{Lu}$ is often found in glycosylated form in many types of plants, including fruits, vegetables, and medicinal herbs (eg, green pepper, celery, broccoli, and parsley), and especially high amounts are found in peanut hulls and in Reseda luteola L. ${ }^{6}$ In traditional Chinese medicine, plants rich in Lu have been used for treating various diseases, such as hypertension, inflammatory disorders, and cancer. ${ }^{5}$ Recent studies have indicated that Lu possesses multiple biological effects, 

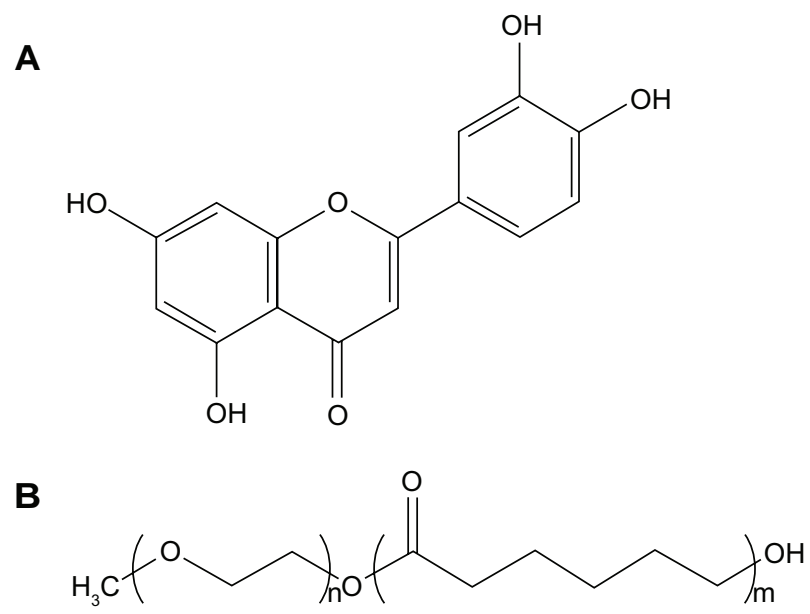

Figure I (A) Molecular structure of luteolin. (B) Molecular structure of monomethoxy poly(ethylene glycol)-poly( $\varepsilon$-caprolactone) copolymer.

such as anti-inflammation, antiallergy, and anticancer, chiefly due to its antioxidant and free radical-scavenging capacities. ${ }^{6-8}$ It was suggested that the number of hydroxyl groups in the structure of flavonoids is directly related to their free radical-scavenging activity. ${ }^{9}$ With four hydroxyl groups, $\mathrm{Lu}$ is highly potent in scavenging free radicals and antioxidants. The anticancer effect of $\mathrm{Lu}$ also is associated with inducing apoptosis, which involves redox regulation, DNA damage, and protein kinases in inhibiting the proliferation of cancer cells and suppressing metastasis and angiogenesis. ${ }^{610-17}$ Moreover, Lu has cytotoxicity in cancer cells or immortalized cells, but not in normal cells, meaning that it has fewer side effects when used in treating cancer. ${ }^{8,12,18}$ Yet Lu has some drawbacks, such as poor water solubility $\left(<2 \times 10^{-2} \mu \mathrm{mol} / \mathrm{mL}\right),{ }^{19}$ low oral absorption, ${ }^{20}$ and bioavailability (30.4\% in rats), ${ }^{21}$ which limit its clinical application. Therefore, it is of interest to promote an injectable aqueous formulation for $\mathrm{Lu}$.

Nanotechnology has the potential to overcome the poor water solubility of lipophilic drugs. ${ }^{22}$ Encapsulation of hydrophobic drugs into nanoparticles is a promising approach to make the drug intravenously injectable. The amphiphilic nanoparticles that comprise a hydrophobic core and a hydrophilic shell are excellent candidates for carrying hydrophobic drugs. Poly( $\varepsilon$-caprolactone)/poly(ethylene glycol) (PCL/PEG) can self-assemble into nanoparticles with core-shell structure: a hydrophobic PCL core and a hydrophilic PEG shell. ${ }^{23}$ While encapsulated with the hydrophobic drug, the hydrophobic PCL segment combined with the drug forms the core and the hydrophilic PEG forms the shell of nanoparticles, making the drug intravenously injectable. ${ }^{24}$ Also, encapsulated with hydrophobic drugs,
PCL/PEG copolymers could enhance stability and systemic circulation $t_{1 / 2}$ of drugs and release drugs at a sustained rate in the optimal range of drug concentration. ${ }^{23,24}$ Moreover, PCL/PEG copolymers are biodegradable, biocompatible, and easy to produce, showing promising applications in drug-delivery systems. ${ }^{25,26}$

Monomethoxy poly(ethylene glycol)-poly(e-caprolactone) (MPEG-PCL) is a diblock PCL/PEG copolymer, and its molecular structure is presented in Figure 1B. Recently, MPEG-PCL micelles were used to deliver hydrophobic drugs (such as honokiol and curcumin), with the goal of creating novel aqueous formulations for these drugs. ${ }^{24,26} \mathrm{In}$ this study, in an attempt to promote an aqueous formulation for $\mathrm{Lu}$, we prepared Lu-encapsulated MPEG-PCL micelles. Our results indicated that encapsulation of Lu in MPEGPCL micelles rendered Lu completely dispersible in water. Also, Lu/MPEG-PCL micelles were able to slowly release Lu and efficiently inhibit the growth of cancer cells in vitro. These data suggested that Lu/MPEG-PCL micelles may be a novel nanoformulation of $\mathrm{Lu}$ with promising application in cancer therapy.

\section{Materials and methods Materials}

Lu was purchased from Skyherb (Huangzhong, People's Republic of China). MPEG-PCL diblock copolymer with a designed molecular weight of 4000 was prepared according to our previous report. ${ }^{26}$ Sprague Dawley rats (weight of $200 \pm 20 \mathrm{~g}$ ) were purchased from the Laboratory Animal Center of Sichuan University (Chengdu, People's Republic of China). The 4T1 breast cancer cells and $\mathrm{C}-26$ colon carcinoma cells were purchased from the ATCC (American Type Culture Collection, Rockville, MD, USA). All studies involving rats were approved by the institute's animal care and use committee.

\section{Preparation of Lu/MPEG-PCL micelles}

Lu/MPEG-PCL micelles were prepared by a self-assembled method. Briefly, Lu and MPEG-PCL diblock copolymer mixture were codissolved in $5 \mathrm{~mL}$ of acetone first; next, the solution was evaporated in a rotator evaporator. Then, $5 \mathrm{~mL}$ of normal saline was added, followed by shaking gently in a $55^{\circ} \mathrm{C}$ water bath. In this process, MPEG-PCL self-assembled into core-shell-structured micelles with core-encapsulated Lu. The Lu-loaded MPEG-PCL micelle solution was centrifuged at $13 \times 10^{3} \mathrm{rpm}$ for 2 minutes to remove the insoluble drugs. Finally, the prepared Lu/MPEG-PCL micelles were lyophilized and stored at $4^{\circ} \mathrm{C}$. 


\section{Characterization of Lu/MPEG-PCL micelles}

The particle size and zeta potential of Lu/MPEG-PCL micelles were obtained from three repeat measurements by a dynamic laser-diffraction particle-size detector and a Malvern Zeta analyzer (Nano-ZS; Malvern Instruments, Malvern, UK), respectively. During the measuring process, the temperature was kept at $25^{\circ} \mathrm{C}$.

Morphology of the Lu/MPEG-PCL micelles was observed under a transmission electron microscope (H-6009IV; Hitachi, Tokyo, Japan): micelles were dissolved with distilled water and placed on a copper grid covered with nitrocellulose. Samples were negatively stained with phosphotungstic acid and dried at room temperature.

The concentration of Lu was determined by high-performance liquid chromatography (HPLC) (Alliance 2695; Waters, Milford, MA, USA). Chromatographic separations were performed on a reversed-phase $\mathrm{C} 18$ column $(250 \times 4.6 \mathrm{~mm}, 5 \mu \mathrm{m}$, SunFire Analysis column; Waters), with a mobile phase composed of methanol and $0.2 \%$ phosphoric acid aqueous solution $(58: 42, \mathrm{v} / \mathrm{v})$ at a flow rate of $1.0 \mathrm{~mL} /$ minute (injected volume, $20 \mu \mathrm{L}$ ). The detection wavelength and the column temperature were set at $360 \mathrm{~nm}$ and $30^{\circ} \mathrm{C}$, respectively. The amount of $\mathrm{Lu}$ was estimated from standard curves obtained by analysis of various doses of a standard substance.

To measure drug loading (DL) and encapsulation efficiency (EE) of Lu/MPEG-PCL micelles, $0.1 \mathrm{~mL}$ of Lu/ MPEG-PCL micelle solution was placed in a preweighed Eppendorf tube and lyophilized to constant weight. Afterwards, the precipitate was dissolved in $0.1 \mathrm{~mL}$ acetone and was diluted by methanol to a total volume of $4 \mathrm{~mL}$. The amount of $\mathrm{Lu}$ in the solution was assayed using HPLC. Finally, the DL and EE of Lu-loaded MPEG-PCL micelles were calculated according to equations (1) and (2):

$$
\begin{gathered}
\mathrm{DL}=\frac{\text { Drug }}{\text { Polymer }+ \text { Drug }} \times 100 \% \\
\mathrm{EE}=\frac{\text { Experimental drug loading }}{\text { Theoretical drug loading }} \times 100 \%
\end{gathered}
$$

\section{In vitro release study}

To determine the release profile of $\mathrm{Lu}$ from Lu/MPEG-PCL micelles, $1 \mathrm{~mL}$ of Lu/MPEG-PCL micelle water solution and Lu in dimethyl sulfoxide solution (as control) at a concentration of $1 \mathrm{mg} / \mathrm{mL}$ was placed in a dialysis bag with a molecular weight cutoff of $7 \mathrm{kDa}$. These dialysis bags were incubated in $30 \mathrm{~mL}$ of phosphate-buffered saline (pH 7.4) containing Tween- $80(0.5 \%, \mathrm{v} / \mathrm{v})$ at $37^{\circ} \mathrm{C}$ with gentle shaking. At predetermined time points, the incubation medium was changed for fresh incubation medium. At each data point, the incubation medium from these two samples was collected separately, the amount of released drug in them was quantified by HPLC, and the accumulated release profile with time was demonstrated. This study was repeated three times, and the results were expressed as mean values \pm standard deviation.

\section{In vivo pharmacokinetics study}

Rats were subjected to jugular venous cannulation 24 hours prior to assays. Under chloral hydrate anesthesia, $2 \mathrm{~cm}$ of the cannula was introduced into the jugular vein toward the heart. The inside of the implanted cannula remained permanently filled with heparin solution, and the free end of the cannula was closed with a plug. These rats were fasted overnight prior to drug administration, and were divided into two groups (three rats in each group): an Lu-treatment group and an Lu/ MPEG-PCL micelle-treatment group. Free Lu was dissolved in Cremophor EL (polyoxyl 35 castor oil) and alcohol (1:1, v/v) solution, followed by dilution with normal saline. Lu/ MPEG-PCL micelles were dissolved in normal saline, followed by intravenous administration of $30 \mathrm{mg} / \mathrm{kg}$ of free $\mathrm{Lu}$ or micelle-encapsulated Lu (Lu/MPEG-PCL micelles). The blood was collected from the jugular venous cannula at different time intervals. Plasma was separated, and $\mathrm{Lu}$ was extracted from plasma with acetonitrile; supernatant fluid was collected and evaporated to dryness by nitrogen-blowing. The dry residues were dissolved in methanol for HPLC analysis. Peak concentration $\left(\mathrm{C}_{\max }\right)$ and time to reach $\mathrm{C}_{\text {max }}$ $\left(T_{\max }\right)$ values were recorded directly from the measured data. Then, $t_{1 / 2}$, area under the curve (AUC) $)_{0-t}$, and $\mathrm{AUC}_{0-\infty}$ values were calculated. Drug and Statistics (DAS) software (version 2.1.1; Mathematical Pharmacology Professional Committee of China), was used for pharmacokinetic analysis.

\section{Cytotoxicity study of Lu/MPEG-PCL micelles in vitro}

The cytotoxicity of free Lu or MPEG-PCL-encapsulated Lu to tumor cell lines was evaluated by 3-(4,5-dimethylthiazol-2yl)-2,5-diphenyltetrazolium bromide (MTT) assay. The 4T1 (breast) and C26 (colon) tumor cell lines were maintained in Roswell Park Memorial Institute 1640 culture medium with $10 \%$ fetal bovine serum and incubated at $37^{\circ} \mathrm{C}$ in a humidified atmosphere ( $95 \%$ air and $5 \% \mathrm{CO}_{2}$ ). The $4 \mathrm{~T} 1$ or $\mathrm{C} 26$ cells were plated at a density of $5 \times 10^{3}$ cells per well in $100 \mu \mathrm{L}$ of culture medium in 96-well plates, and were incubated at 

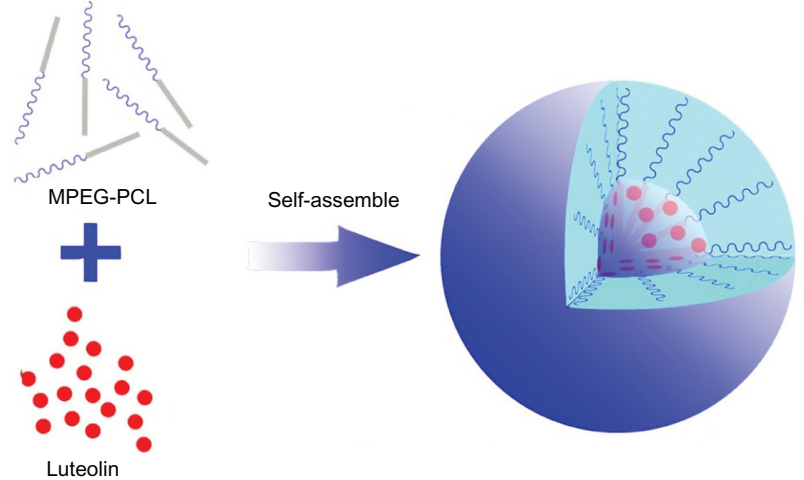

Figure 2 Preparation scheme of luteolin/monomethoxy poly(ethylene glycol)poly( $\varepsilon$-caprolactone) (MPEG-PCL) micelles by a self-assembly method. Luteolin and MPEG-PCL were codissolved in acetone followed by evaporation of the acetone. Then, normal saline was added making the self-assembly of the core-shell-structured micelles with core-encapsulated luteolin.

$37^{\circ} \mathrm{C}$ for 24 hours. Cells were then treated for 48 hours with different concentrations of free Lu or micelle-encapsulated Lu or blank MPEG-PCL micelles. Control cultures were treated with $0.1 \%$ dimethyl sulfoxide; this concentration was not found to affect cell growth. The effect of Lu on cell viability was determined using MTT, a colorimetric assay with the reduction of MTT as the assessable end point. Data were shown as proportional to cell viability (\%) by comparing the treated group with the untreated cells.

\section{Statistical analysis}

Data are expressed as mean values \pm standard deviation. Statistical analysis was performed with unpaired Student's $t$-tests. $P$-values less than 0.05 were considered to be statistically significant.

\section{Results}

\section{Preparation and characterization of Lu/MPEG-PCL micelles}

Lu/MPEG-PCL micelles were prepared by a self-assembly method; a schematic diagram is shown in Figure 2. Altered ratios of Lu and MPEG-PCL diblock copolymer were codissolved in $5 \mathrm{~mL}$ of acetone. Then the acetone was evaporated under vacuum in a rotary evaporator, leaving a layer of pale-yellow transparent membranous substances adhering to the inside wall of the glass container. Then, $5 \mathrm{~mL}$ of normal saline was added, followed by shaking in a $55^{\circ} \mathrm{C}$ water bath until the membranous substances were completely dissolved. In this process, the hydrophobic Lu was encapsulated into the core of the MPEG-PCL micelles, creating Lu/MPEG-PCL micelles. To optimize the process parameters, different Lu/MPEG-PCL mass ratios in the feed were studied in our work, and results are presented in Table 1. With the increase in Lu/micelle ratio, drug loading increased, but encapsulation efficiency decreased; also, particle size and polydispersity index (PDI) increased, but the stability of the Lu/MPEG-PCL micelles decreased. When the Lu/ MPEG-PCL mass ratio was $\geq 5 / 95$, the obtained Lu/MPEGPCL micelles were not stable in water (micelles tended to aggregate, forming aggregates); thus, the Lu/MPEG-PCL mass ratio at 4/96 was chosen in our study.

Sample III was characterized in detail and used for future applications. Drug loading and encapsulation efficiency of Lu/MPEG-PCL micelles were $3.93 \% \pm 0.25 \%$ and $98.32 \% \pm 1.12 \%$, respectively. Moreover, these Lu/MPEGPCL micelles had a median particle size of $38.6 \pm 0.6 \mathrm{~nm}$ (PDI $=0.16 \pm 0.02 \mathrm{~nm}$ ) and a mean slight negative surface charge of $-3.54 \pm 0.32 \mathrm{mV}$. The particle-size distribution spectrum of freshly prepared Lu/MPEG-PCL micelles is presented in Figure 3A, showing that Lu/MPEG-PCL micelles had a narrow particle-size distribution. The zeta-potential distribution spectrum of Lu/MPEG-PCL micelles indicated that Lu/MPEG-PCL micelles had a slight negative surface charge (shown in Figure 3B).

After Lu/MPEG-PCL micelles were freeze-dried, the resolubility of these lyophilized Lu/MPEG-PCL micelles was examined. As shown in Figure 3C, these redissolved micelles had a mean particle size of $45.8 \pm 2.2 \mathrm{~nm}(\mathrm{PDI}=0.15 \pm 0.04)$. These redissolved micelles had a mean slight negative surface charge of $-4.75 \pm 0.68 \mathrm{mV}$, and the zeta-potential distribution

Table I Properties of luteolin-loaded monomethoxy poly(ethylene glycol)-poly(E-caprolactone) (MPEG-PCL) micelles

\begin{tabular}{|c|c|c|c|c|c|c|}
\hline Sample & $\begin{array}{l}\text { Luteolin/MPEG- } \\
\text { PCL mass ratio }\end{array}$ & DL (\%) & EE (\%) & $\begin{array}{l}\text { Mean size } \\
(\mathrm{nm})\end{array}$ & PDI & Stability \\
\hline I & $0 / 100$ & 0 & 0 & $23.4 \pm 0.2$ & $0.10 \pm 0.01$ & Yes \\
\hline II & $2 / 98$ & $1.99 \pm 0.01$ & $99.50 \pm 0.50$ & $24.5 \pm 0.3$ & $0.12 \pm 0.01$ & Yes \\
\hline III & $4 / 96$ & $3.93 \pm 0.25$ & $98.32 \pm 1.12$ & $38.6 \pm 0.6$ & $0.16 \pm 0.02$ & Yes \\
\hline IV & $5 / 95$ & $4.72 \pm 0.07$ & $94.49 \pm 1.31$ & $43.7 \pm 0.1$ & $0.18 \pm 0.02$ & No \\
\hline V & $6 / 94$ & $5.55 \pm 0.05$ & $92.56 \pm 0.84$ & $46.6 \pm 0.2$ & $0.23 \pm 0.04$ & No \\
\hline
\end{tabular}

Abbreviations: EE, encapsulation efficiency; DL, drug loading; PDI, polydispersity index; nm, nanometer. 

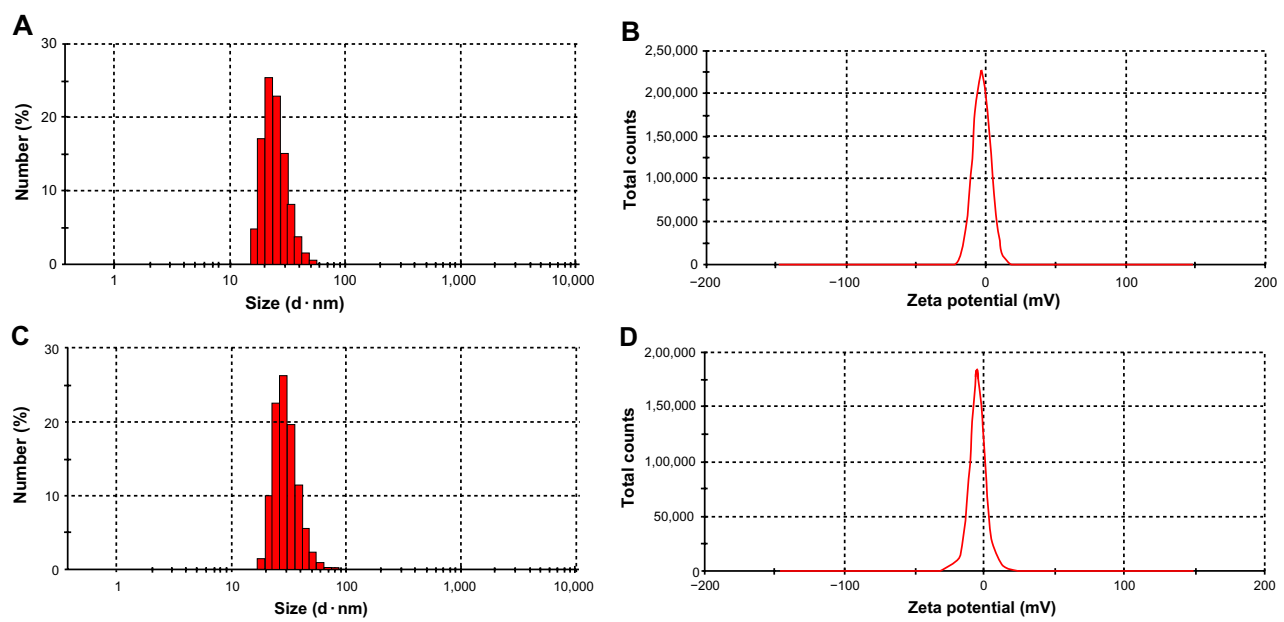

Figure 3 (A-D) Particle-size distribution and zeta-potential distribution spectra of luteolin (Lu)/monomethoxy poly(ethylene glycol)-poly(E-caprolactone) (MPEG-PCL) micelles. (A and B) Particle-size distribution spectrum and zeta-potential distribution spectrum of freshly prepared Lu/MPEG-PCL micelles, respectively; (C and D) particle-size distribution spectrum and zeta-potential distribution spectrum of redissolved Lu/MPEG-PCL micelles after freeze-drying, respectively. Each test was repeated three times.

spectrum is shown in Figure 3D. The morphology of the Lu/ MPEG-PCL micelles, determined by transmission electron microscopy, is shown in Figure 4, revealing that Lu/MPEGPCL micelles were uniform and spherical, with a diameter of $\sim 30 \mathrm{~nm}$.

The appearance of the Lu/MPEG-PCL micelle aqueous solution is shown in Figure 5. Pure Lu could not be dissolved in pure water (Figure 5D), as confirmed by the observation of turbid oyster white slurry. However, Lu/MPEG-PCL micelle solution loaded with an equivalent quantity of Lu was transparent (Figure 5B), indicating the drug-loaded micelles

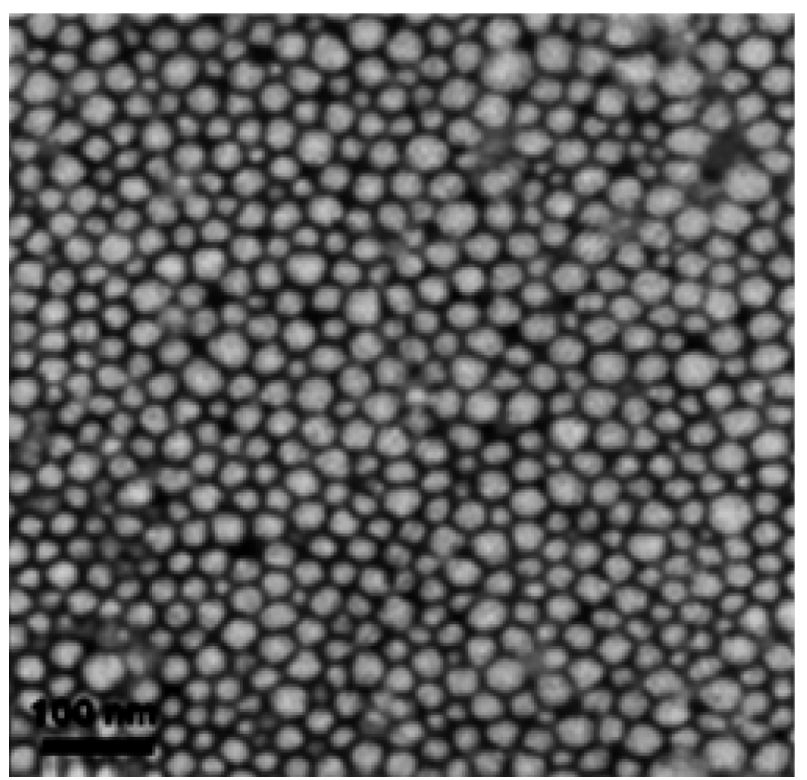

Figure 4 Transmission electron microscopy image of luteolin/monomethoxy poly(ethylene glycol)-poly( $\varepsilon$-caprolactone) micelles. were fully dispersible in water. Freeze-dried Lu/MPEG-PCL micelles (Figure 5C) were also fully dispersible in water. Thus, we can draw a conclusion that encapsulation of $\mathrm{Lu}$ in MPEG-PCL micelles renders Lu completely dispersible in aqueous media, making Lu intravenously injectable.

\section{In vitro release profile}

The in vitro release behavior of MPEG-PCL micelle-encapsulated Lu and free Lu in phosphate-buffered saline ( $\mathrm{pH} 7.4$ ) at $37^{\circ} \mathrm{C}$ was studied using a dialysis method, and the results are shown in Figure 6. Free Lu was observed to be rapidly released and reached its peak of $82.8 \%$ of the total in the first 12 hours. In comparison, MPEG-PCL micelle-encapsulated Lu showed a two-phase release profile. A relatively rapid release of MPEG-PCL micelle-encapsulated $\mathrm{Lu}$ in the first

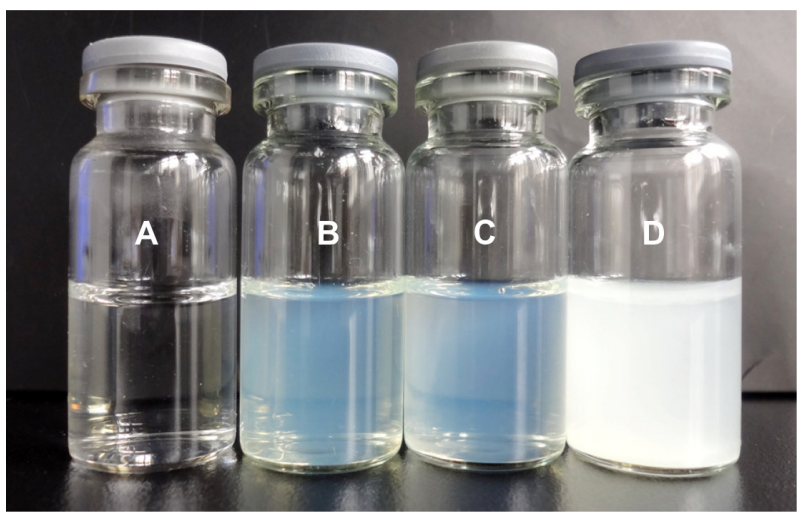

Figure 5 (A-D) Luteolin (Lu)/monomethoxy poly(ethylene glycol)-poly( $\varepsilon$ caprolactone) (MPEG-PCL) micelles render Lu completely dispersible in aqueous media. (A) Water as control; (B) freshly prepared Lu/MPEG-PCL micelle solution; (C) redissolved Lu/MPEG-PCL micelles after freeze-drying; (D) pure Lu in water. 


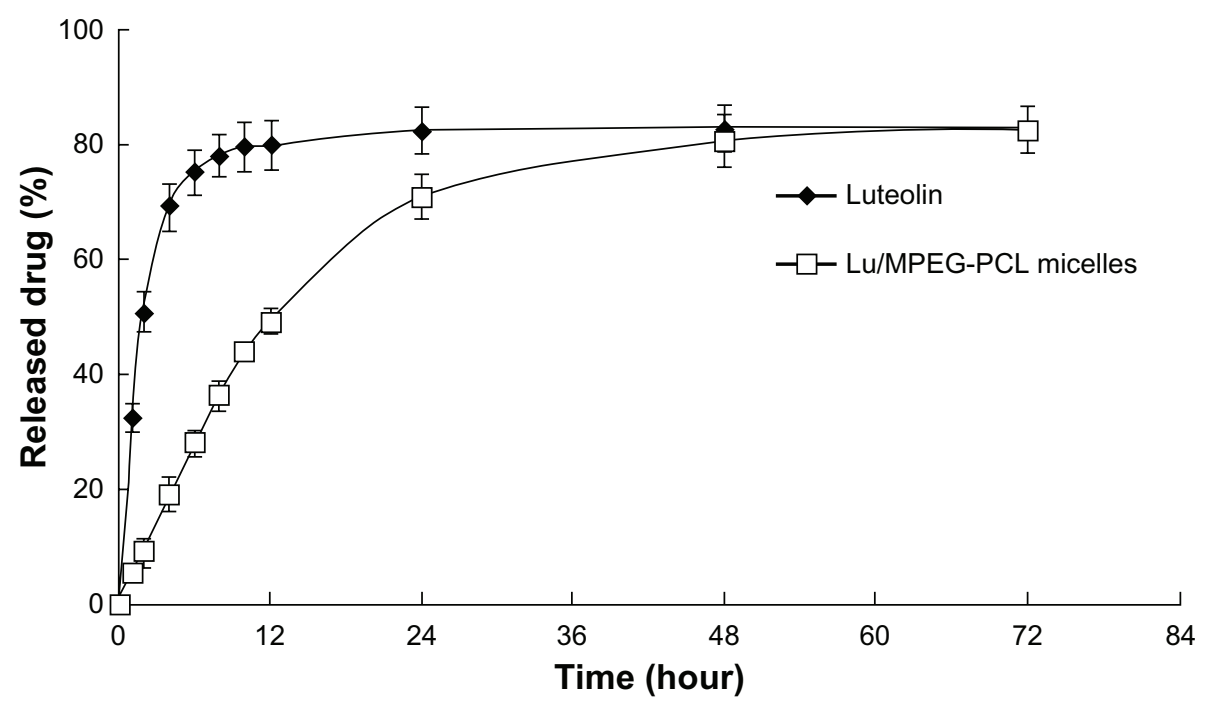

Figure 6 In vitro release profile of luteolin (Lu)/monomethoxy poly(ethylene glycol)-poly( $\varepsilon$-caprolactone) (MPEG-PCL) micelles. The in vitro release profiles were examined using a dialysis method. Lu could be slowly released from micelles in vitro. The test was repeated three times.

phase was observed in the first 12 hours $(49.3 \%)$, followed by a sustained and slower release phase. About $82.5 \%$ of Lu was released from Lu-encapsulated MPEG-PCL micelles in 72 hours. The results indicated that Lu could be slowly released from micelles in a sustained manner in vitro. The sustained release of Lu from MPEG-PCL micelles might be due to two reasons: (1) the diffusion of Lu from the micelles to the medium, and (2) degradation and hydrolysis of micelleinduced release of $\mathrm{Lu}$ to the medium.

\section{In vivo pharmacokinetics analysis}

To confirm whether Lu/MPEG-PCL micelles can improve the pharmacokinetics of Lu in vivo, the pharmacokinetics of free Lu and Lu/MPEG-PCL micelles was studied in rats. Rats were intravenously administered with Lu or Lu/MPEG-PCL micelles ( $\mathrm{Lu} 30 \mathrm{mg} / \mathrm{kg}$ ). Blood was collected at different time intervals. Results of pharmacokinetics are shown in Table 2 and Figure 7 and were analyzed by DAS software. For MPEG-PCL micelle-encapsulated Lu, $\mathrm{T}_{\max }, t_{1 / 2}, \mathrm{AUC}_{0-\mathrm{t}}$, $\mathrm{AUC}_{0-\infty}$, and $\mathrm{C}_{\max }$ were 5 minutes, $152.25 \pm 49.92$ minutes, $2914.05 \pm 445.17 \mathrm{mg} / \mathrm{L} /$ minute, $2989.03 \pm 433.22 \mathrm{mg} / \mathrm{L} /$ minute, and $92.70 \pm 11.61 \mathrm{mg} / \mathrm{L}$, respectively. For pure $\mathrm{Lu}, \mathrm{T}_{\max }, t_{1 / 2}, \mathrm{AUC}_{0-\mathrm{t}}, \mathrm{AUC}_{0-\infty}$, and $\mathrm{C}_{\max }$ were 5 minutes, $7.16 \pm 1.23$ minutes, $502.65 \pm 140.12 \mathrm{mg} / \mathrm{L} / \mathrm{minute}$
$503.81 \pm 141.41 \mathrm{mg} / \mathrm{L} /$ minute, and $38.98 \pm 7.73 \mathrm{mg} / \mathrm{L}$, respectively. Thus, it was suggested that encapsulation of $\mathrm{Lu}$ in MPEG-PCL micelles improved $t_{1 / 2}(P=0.007, \mathrm{Lu} / \mathrm{MPEG}-$ PCL versus [vs] Lu), $\mathrm{AUC}_{0-\mathrm{t}}(P=0.001, \mathrm{Lu} / \mathrm{MPEG}-\mathrm{PCL}$ vs $\mathrm{Lu}), \mathrm{AUC}_{0-\infty}(P=0.001$, Lu/MPEG-PCL vs Lu $)$, and $\mathrm{C}_{\max }$ $(P=0.003, \mathrm{Lu} / \mathrm{MPEG}-\mathrm{PCL}$ vs $\mathrm{Lu})$ of Lu in vivo.

\section{In vitro cytotoxicity of Lu/ MPEG-PCL micelles}

The cytotoxicity of Lu/MPEG-PCL micelles was compared with that of pure Lu on 4T1 breast cancer cells and C26 colon carcinoma cells in vitro. After cancer cells were treated with micelle-encapsulated Lu, pure Lu or blank MPEG-PCL micelles for 48 hours, cell viability was measured by MTT assay. As shown in Figure 8, the blank MPEG-PCL micelles did not show any cytotoxicity activity in 4T1 cancer cells or C26 cancer cells, while both pure Lu and Lu/MPEG-PCL micelles showed significant cytotoxic activity in both of these two cell lines. Even the cytotoxicity of MPEG-PCL micelle-encapsulated $\mathrm{Lu}$ was stronger than that of free Lu. The half-maximal inhibitory concentrations for free Lu and MPEG-PCL micelle-encapsulated Lu on 4T1 cells were $10.21 \pm 1.64 \mu \mathrm{g} / \mathrm{mL}$ and $6.4 \pm 2.30 \mu \mathrm{g} / \mathrm{mL}$, respectively, and those in C26 cells were $13.06 \pm 1.84 \mu \mathrm{g} / \mathrm{mL}$ and

Table 2 Pharmacokinetic parameters for both formulations

\begin{tabular}{llllll}
\hline Groups & $\mathbf{T}_{\max }$ minutes & $\boldsymbol{t}_{1 / 2}$ minutes & AUC $_{(0-\mathrm{t})} \mathbf{m g} / \mathrm{L} / \mathbf{m i n u t e}$ & AUC $_{(0-\infty)} \mathbf{m g} / \mathrm{L} / \mathbf{m i n u t e}$ & $\mathbf{C}_{\max } \mathbf{m g} / \mathbf{L}$ \\
\hline Luteolin & 5 & $7.16 \pm 1.23$ & $502.65 \pm 140.12$ & $503.81 \pm 141.41$ & $38.98 \pm 7.73$ \\
Lu/MPEG-PCL & 5 & $152.25 \pm 49.92$ & $2914.05 \pm 445.17$ & $2989.03 \pm 433.22$ & $92.70 \pm 11.61$ \\
$P$ & - & 0.007 & 0.001 & 0.001 & 0.003 \\
\hline
\end{tabular}

Abbreviations: $T_{\max }$, time to reach $C_{\max } ; t_{1 / 2}$, half-life; AUC, area under the curve; $C_{\max }$, peak concentration; MPEG-PCL, monomethoxy poly(ethylene glycol)-poly( $\varepsilon$-caprolactone). 


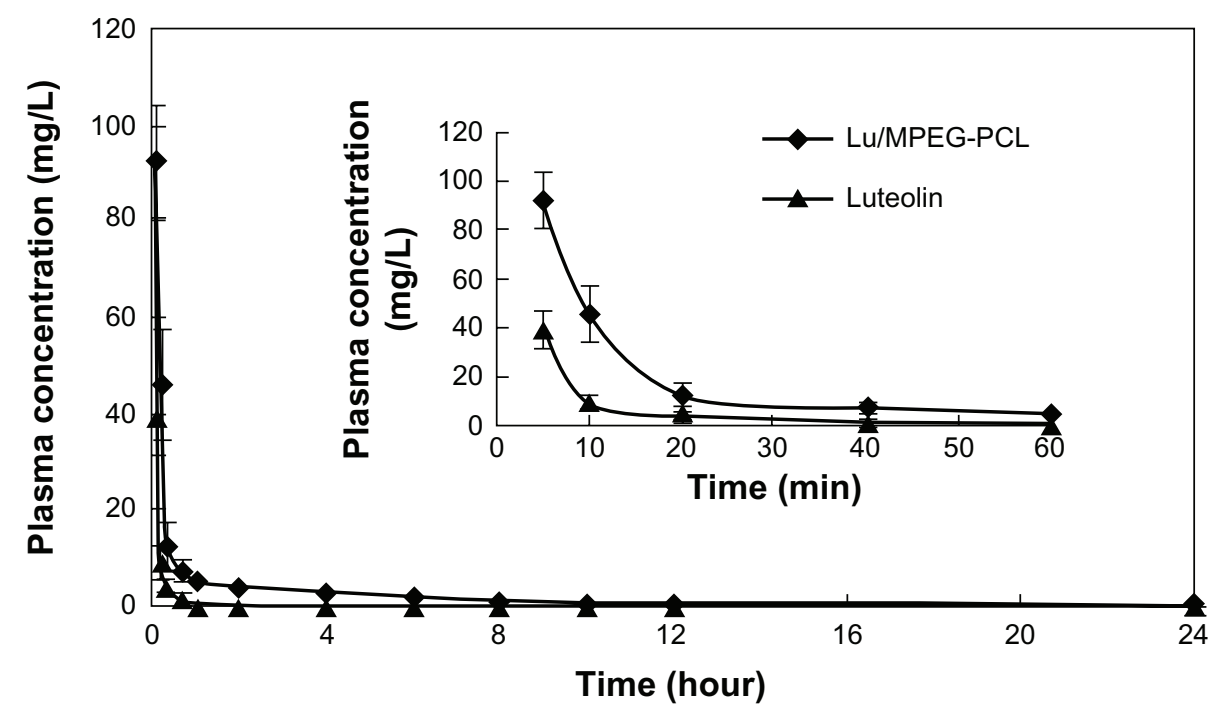

Figure 7 In vivo pharmacokinetics assays of luteolin (Lu)/monomethoxy poly(ethylene glycol)-poly(E-caprolactone) (MPEG-PCL) micelles. Encapsulation of luteolin in MPEGPCL micelles improved the pharmacokinetics of Lu. The test was repeated three times.

$12.62 \pm 2.17 \mu \mathrm{g} / \mathrm{mL}(P>0.05)$, respectively. This result indicated that MPEG-PCL micelle encapsulated-Lu as well as free Lu still had anticancer activity.

\section{Discussion}

To overcome the poor water-solubility of Lu, we encapsulated $\mathrm{Lu}$ into MPEG-PCL micelles by a self-assembly method in this study, producing Lu/MPEG-PCL micelles. The Lu/ MPEG-PCL micelles were about $40 \mathrm{~nm}$ in diameter, rendering Lu completely dispersible in water (Figure 5). Also, Lu/ MPEG-PCL micelles showed drug loading of $3.93 \% \pm 0.25 \%$ and a high encapsulation efficiency of $98.32 \% \pm 1.12 \%$, making this an aqueous formulation of $\mathrm{Lu}$. In addition, the $\mathrm{Lu} / \mathrm{MPEG}-\mathrm{PCL}$ micelles had a slight negative surface charge of $-3.54 \pm 0.32 \mathrm{mV}$, which increased the circulation time of the drug. Surface charge is important in determining whether the nanoparticles will cluster in blood flow or will adhere to or interact with oppositely charged cell membrane. ${ }^{27}$ The plasma and blood cells always had a negative charge; nanoparticles with slight negative surface charge may minimize nonspecific interaction with these components through electrostatic interactions. ${ }^{28-30}$ The Lu/MPEG-PCL micelles were able to slowly release Lu (Figure 6), indicating that Lu-encapsulated MPEG-PCL micelles may enhance systemic circulation $t_{1 / 2}$ of Lu in vivo. The sustained release of Lu from MPEG-PCL micelles might be due to the diffusion of Lu from micelles and the degradation or hydrolysis of micelles. Pharmacokinetics in rats was studied, and the results (Figure 7) proved that $\mathrm{Lu} / \mathrm{MPEG}-\mathrm{PCL}$ micelles enhance systemic circulation $t_{1 / 2}$ of $\mathrm{Lu}$ in vivo. Meanwhile, the Lu/MPEG-PCL micelles
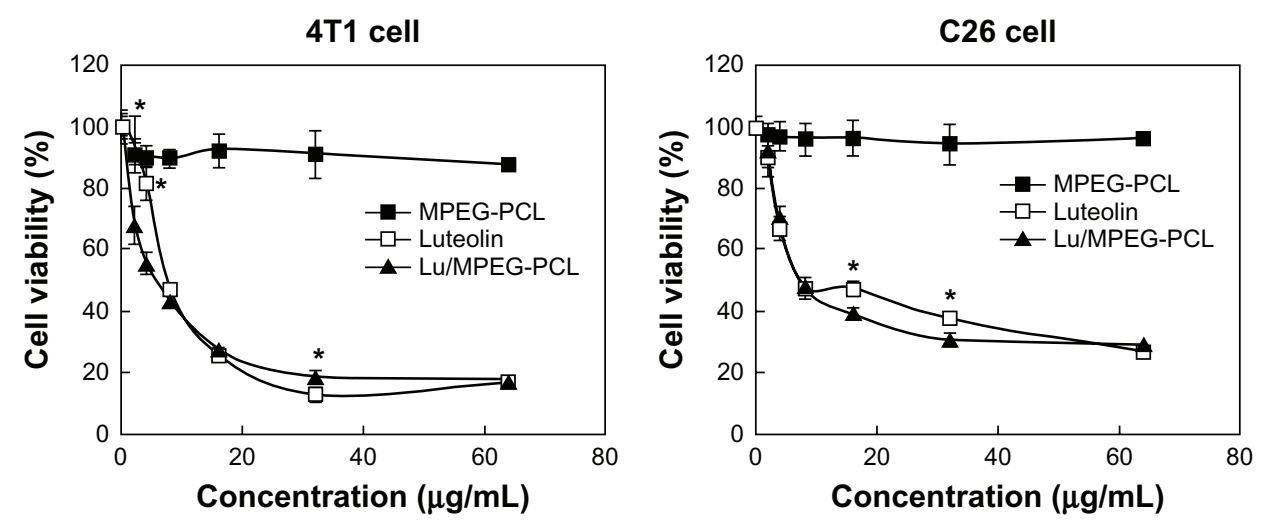

Figure 8 Cytotoxicity of luteolin (Lu)/monomethoxy poly(ethylene glycol)-poly(E-caprolactone) (MPEG-PCL) micelles on cancer cells in vitro. As well as free Lu, Lu/MPEGPCL micelles could efficiently inhibit the viability of both 4TI breast cancer cells and C26 colon cancer cells, while blank MPEG-PCL micelles had little cytotoxicity on these cells. The test was repeated three times.

Notes: *significant difference between luteolin and Lu/MPEGPCL. 
maintained the cytotoxicity of $\mathrm{Lu}$, which showed in an in vitro cytotoxicity study in 4T1 and C26 cells (Figure 8). The $\mathrm{IC}_{50}$ values for pure Lu and MPEG-PCL micelle-encapsulated Lu in $4 \mathrm{~T} 1$ cells were $10.21 \pm 1.64 \mu \mathrm{g} / \mathrm{mL}$ and $6.4 \pm 2.30 \mu \mathrm{g} / \mathrm{mL}$, respectively, and those in C26 cells were $13.06 \pm 1.84 \mu \mathrm{g} / \mathrm{mL}$ and $12.62 \pm 2.17 \mu \mathrm{g} / \mathrm{mL}$, respectively.

$\mathrm{Lu}$ is a promising agent for anticancer therapy. But $\mathrm{Lu}$ is a lipophilic drug and insoluble in water, which limits its clinical application. Encapsulation by nanoparticles appears to be a promising approach to overcome the hydrophobicity of drugs. There are many encapsulation methods, such as spray-drying, solvent removal, etc, to encapsulate $\mathrm{Lu}$ into micelles or liposomes. ${ }^{31}$ Also, inclusion complexation with cyclodextrin could enhance the water solubility of $\mathrm{Lu} .^{32,33}$ In this work, we used MPEG-PCL micelles to encapsulate Lu. In the preparation process, Lu and MPEG-PCL diblock copolymer mixture was first dissolved in acetone solution, followed by evaporating the organic solvent. Then, the amphiphilic MPEG-PCL copolymers self-assembled into supramolecular arrangements possessing a hydrophobic inner core and a hydrophilic shell in water, and Lu self-assembled into the hydrophobic core of the micelles because of its hydrophobicity. The preparation procedure was simple and easy to scale up. These MPEG-PCL micelles are biodegradable, biocompatible, amphiphilic, stable in blood, nontoxic, nonimmunogenic, non-inflammatory, and small in size; this makes MPEG-PCL micelles an excellent candidate for drugdelivery systems. ${ }^{27}$ Thus, MPEG-PCL micelle-encapsulated Lu might be an interesting formulation.

In summary, MPEG-PCL micelles were used to encapsulate $\mathrm{Lu}$, creating Lu/MPEG-PCL micelles. The Lu/MPEGPCL micelles improved the water solubility, enhanced circulation $t_{1 / 2}$ in vivo, and maintained the cytotoxicity of Lu. Lu/MPEG-PCL micelles are an injectable aqueous formulation of $\mathrm{Lu}$ and may have potential application in cancer treatment.

\section{Conclusion}

MPEG-PCL micelle-encapsulated Lu was prepared, with the goal of improving the water solubility of Lu. These $\mathrm{Lu} / \mathrm{MPEG}-\mathrm{PCL}$ micelles can slowly release $\mathrm{Lu}$, enhance systemic circulation $t_{1 / 2}$ of $\mathrm{Lu}$ in vivo, and inhibit the growth of cancer cells in vitro, showing promising application in cancer treatment.

\section{Acknowledgments}

This work was supported by the Specialized Research Fund for the Doctoral Program of Higher Education
(20110181120087), the National Natural Science Foundation of China (81201785), the National Science and Technology Major Project (2013ZX09301-304-008), the Key Technologies R\&D program of Sichuan Province (2013SZ0048), and the National Key Basic Research Program (973) of China (2010CB529900).

\section{Disclosure}

The authors report no conflicts of interest in this work.

\section{References}

1. Jemal A, Bray F, Center M, Ferlay J, Ward E, Forman D. Global cancer statistics. CA Cancer J Clin. 2011;61(2):69-90.

2. Newman DJ, Cragg GM. Natural products as sources of new drugs over the last 25 years. J Nat Prod. 2007;70(3):461-477.

3. Newman DJ. Natural products as leads to potential drugs: an old process or the new hope for drug discovery? J Med Chem. 2008;51(9): 2589-2599.

4. Harvey AL. Natural products in drug discovery. Drug Discov Today. 2008;13(19-20):894-901.

5. Harborne JB, Williams CA. Advances in flavonoid research since 1992. Phytochemistry. 2000;55(6):481-504.

6. Lin Y, Shi R, Wang X, Shen HM. Luteolin, a flavonoid with potential for cancer prevention and therapy. Curr Cancer Drug Targets. 2008;8(7): 634-646.

7. Seelinger G, Merfort I, Wölfle U, Schempp CM. Anti-carcinogenic effects of the flavonoid luteolin. Molecules. 2008;13(10):2628-2651.

8. Nijveldt RJ, van Nood E, van Hoorn DEC, Boelens PG, van Norren K, van Leeuwen PAM. Flavonoids: a review of probable mechanisms of action and potential applications. The Am J Clin Nutr. 2001;74(4): 418-425.

9. Ueda H, Yamazaki C, Yamazaki M. Luteolin as an anti-inflammatory and anti-allergic constituent of Perilla frutescens. Biol Pharm Bull. 2002;25(9):1197-1202.

10. Attoub S, Hassan AH, Vanhoecke B, et al. Inhibition of cell survival, invasion, tumor growth and histone deacetylase activity by the dietary flavonoid luteolin in human epithelioid cancer cells. Eur J Pharmacol. 2011;651(1-3):18-25.

11. Yang SF, Yang WE, Chang HR, Chu SC, Hsieh YS. Luteolin induces apoptosis in oral squamous cancer cells. J Dent Res. 2008;87(4): 401-406.

12. Horinaka M, Yoshida T, Shiraishi T, et al. Luteolin induces apoptosis via death receptor 5 upregulation in human malignant tumor cells. Oncogene. 2005;24(48):7180-7189.

13. Byun S, Lee KW, Jung SK, et al. Luteolin inhibits protein kinase $C \varepsilon$ and c-Src activities and UVB-induced skin cancer. Cancer Res. 2010;70(6): 2415-2423.

14. Jang S, Kelley KW, Johnson RW. Luteolin reduces IL-6 production in microglia by inhibiting JNK phosphorylation and activation of AP-1. Proc Natl Acad Sci U SA. 2008;105(21):7534-7539.

15. Zhou Q, Yan B, Hu X, Li XB, Zhang J, Fang J. Luteolin inhibits invasion of prostate cancer PC3 cells through E-cadherin. Mol Cancer Ther. 2009;8(6):1684-1691.

16. Lee WJ, Wu LF, Chen WK, Wang CJ, Tseng TH. Inhibitory effect of luteolin on hepatocyte growth factor/scatter factor-induced HepG2 cell invasion involving both MAPK/ERKs and PI3K-Akt pathways. Chem Biol Interact. 2006;160(2):123-133.

17. Bagli E, Stefaniotou M, Morbidelli L, et al. Luteolin inhibits vascular endothelial growth factor-induced angiogenesis; inhibition of endothelial cell survival and proliferation by targeting phosphatidylinositol 3'-kinase activity. Cancer Res. 2004;64(21):7936-7946. 
18. Samy RP, Gopalakrishnakone P, Ignacimuthu S. Anti-tumor promoting potential of luteolin against 7, 12-dimethylbenz(a)anthraceneinduced mammary tumors in rats. Chem Biol Interact. 2006;164(1-2): $1-14$.

19. Kaminaga Y, Nagatsu A, Akiyama T, et al. Production of unnatural glucosides of curcumin with drastically enhanced water solubility by cell suspension cultures of Catharanthus roseus. FEBS Lett. 2003;555(2): 311-316.

20. Zhou P, Li LP, Luo SQ, Jiang HD, Zeng S. Intestinal absorption of luteolin from peanut hull extract is more efficient than that from individual pure luteolin. J Agric Food Chem. 2008;56(1):296-300.

21. Chen Z, Tu M, Sun S, et al. The exposure of luteolin is much lower than that of apigenin in oral administration of Flos Chrysanthemi extract to rats. Drug Metab Pharmacokinet. 2012;27(1):162-168.

22. Zhang L, Chan JM, Gu FX, et al. Self-assembled lipid-polymer hybrid nanoparticles: a robust drug delivery platform. ACS Nano. 2008;2(8): 1696-1702.

23. Gou ML, Wei XW, Men K, et al. PCL/PEG copolymeric nanoparticles: potential nanoplatforms for anticancer agent delivery. Curr Drug Targets. 2011;12(8):1131-1150.

24. Gou ML, Men K, Shi HS, et al. Curcumin-loaded biodegradable polymeric micelles for colon cancer therapy in vitro and in vivo. Nanoscale. 2011;3(4):1558-1567.

25. Wei XW, Gong CY, Gou ML, et al. Biodegradable poly( $\varepsilon$-caprolactone)poly(ethylene glycol) copolymers as drug delivery system. Int J Pharm. 2009;381(1):1-18.
26. Gou ML, Zheng XL, Men K, et al. Self-assembled hydrophobic honokiol loaded MPEG-PCL diblock copolymer micelles. Pharm Res. 2009;26(9):2164-2173.

27. Kumari A, Yadav SK, Yadav SC. Biodegradable polymeric nanoparticles based drug delivery systems. Colloids Surf B Biointerfaces. 2010;75(1) $1-18$.

28. Turner M, Clough G, Michel C. The effects of cationised ferritin and native ferritin upon the filtration coefficient of single frog capillaries. Evidence that proteins in the endothelial cell coat influence permeability. Microvasc Res. 1983;25(2):205-222.

29. Vink H, Duling BR. Identification of distinct luminal domains for macromolecules, erythrocytes, and leukocytes within mammalian capillaries. Circ Res. 1996;79(3):581-589.

30. Dellian M, Yuan F, Trubetskoy V, Torchilin V, Jain R. Vascular permeability in a human tumour xenograft: molecular charge dependence. $\mathrm{Br}$ J Cancer. 2000;82(9):1513-1518.

31. Munin A, Edwards-Lévy F. Encapsulation of natural polyphenolic compounds; a review. Pharmaceutics. 2011;3(4):793-829.

32. Kwon Y, Kim H, Park S, Jung S. Enhancement of solubility and antioxidant activity of some flavonoids based on the inclusion complexation with sulfobutylether $\beta$-cyclodextrin. Bull Korean Chem Soc. 2010;31(10):3035-3037.

33. Kim H, Jung S. Aqueous solubility enhancement of some flavones by complexation with cyclodextrins. Bull Korean Chem Soc. 2008;29(3) $590-594$.
International Journal of Nanomedicine

\section{Publish your work in this journal}

The International Journal of Nanomedicine is an international, peerreviewed journal focusing on the application of nanotechnology in diagnostics, therapeutics, and drug delivery systems throughout the biomedical field. This journal is indexed on PubMed Central, MedLine, CAS, SciSearch $₫$, Current Contents $₫ /$ Clinical Medicine,

\section{Dovepress}

Journal Citation Reports/Science Edition, EMBase, Scopus and the Elsevier Bibliographic databases. The manuscript management system is completely online and includes a very quick and fair peer-review system, which is all easy to use. Visit http://www.dovepress.com/ testimonials.php to read real quotes from published authors. 